\title{
lieuxdits \#7
}

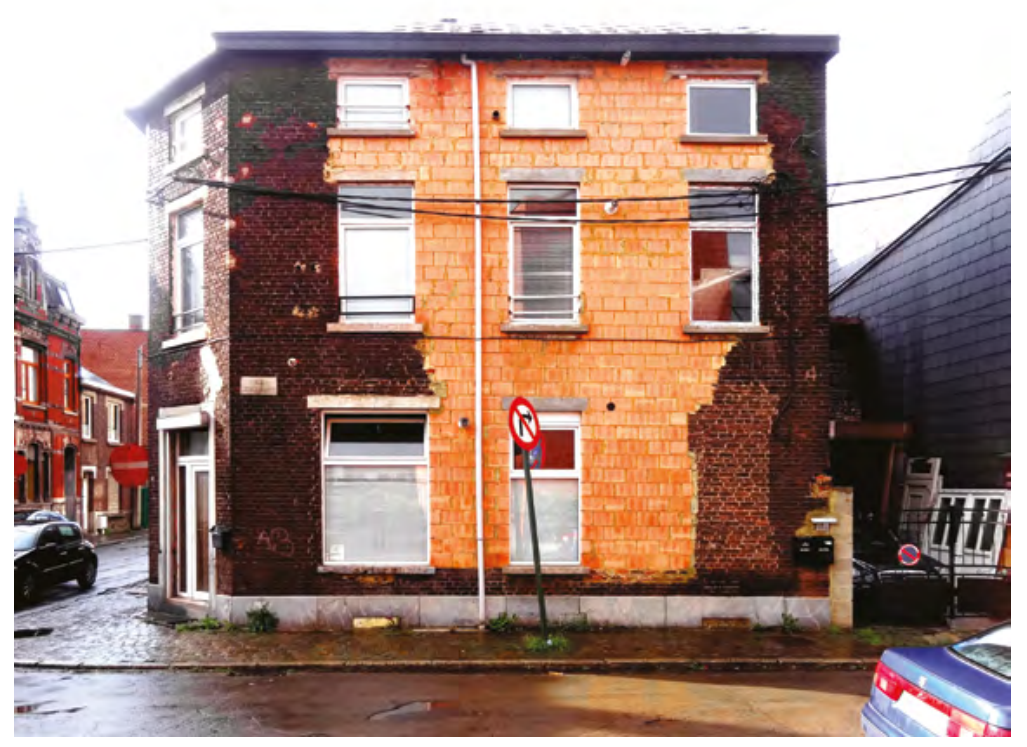

Référence bibliographique :

Damien Claeys, Benoît Cruysmans, Jean-Jacques Jungers, Anna Puigjaner et Marie-Christine Raucent, "Une cité des peintres en Uchronie", lieuxdits\#7, mars 2014, pp.3-6.

\section{La revue lieuxdits}

Faculté d'architecture, d'ingénierie architecturale, d'urbanisme (LOCI)

Univesrité catholique de Louvain (UCL).

Éditeur responsable : Jean-Paul Verleyen, place des Sciences, 1 - 1348 Louvain-la-Neuve

Comité de rédaction : Martin Buysse, Damien Claeys, Gauthier Coton,

Jean-Philippe De Visscher, Guillaume Vanneste, Jean-Paul Verleyen

Conception graphique : Nicolas Lorent

Impression : école d'imprimerie Saint-Luc Tournai

\section{(c) (1) () $\Theta$}




\title{
Une cité des peintres en Uchronie
}

\author{
par Damien Claeys, Benoît Cruysmans, \\ Jean-Jacques Jungers, Anna Puigjaner \\ et Marie-Christine Raucent
}

En Uchronie, deux archéologues belges ont découvert dans un lieu qu'ils préfèrent garder secret une curieuse cité. Il semblerait que celle-ci fût dédiée à la pratique de la peinture, cet art quanjourd'hui seuls quelques initiés perpétuent et qui consiste à représenter ou à exprimer une idée personnelle en appliquant des matières sur une surface choisie.

Les spécificités de chacune des unités constitutives de la cité seraient à cepoint proche des particularités de certaines æuvres picturales connues quélles permettraient, selon les archéologues, d'identifier avec certitude les artistes qui les auraient occupées.

Une énigme reste sans réponse : comment des peintres de périodes différentes auraient-ils pu se retrouver simultanément dans cette cité?

\section{Le contexte pédagogique}

Sur le site de Bruxelles, le dispositif pédagogique des ateliers d'architecture proposé aux étudiants de première année de bachelier - année d'initiation à l'architecture - prévoit quatre projets. Outre les techniques de représentation, ils abordent successivement les dimensions esthétique (la forme), d'usage (la fonction), constructive (la structure) et contextuelle (le lieu). Dans ce projet de cité des peintres, les étudiants sont confrontés, pour la première fois de leur cursus, à un programme constitué de plusieurs fonctions destinées à un usager défini.

Répondre à cette demande n'est pas chose facile. L'exercice est donc organisé en deux temps.

Dans un premier temps, la phase d'analyse, l'étudiant collecte et analyse les données qui vont lui permettre de générer une idée qui le guidera dans la matérialisation de son projet. II expérimente spatialement les mesures utiles pour chacune des fonctions constitutives du programme et il analyse ce dernier pour aboutir à la rédaction d'un scénario.

Dans un second temps, la phase de synthèse, l'étudiant ajuste son idée de manière itérative, utilisant le travail en maquette et le dessin. À partir de son scénario, il matérialise son idée génératrice afin d'obtenir un artefact formant un tout cohérent.

Tout au long de l'exercice, l'enseignant accompagne l'étudiant dans ce processus qui lui est inconnu l'aidant à synthétiser un nombre important d'informations et/ou de contraintes afin d'en extraire une idée qu'il transcrira spatialement. L'étudiant découvre ainsi quelques éléments du jeu architectural que sont l'agencement de fonctions, de matières, d'espaces... et, petit à petit, prend conscience de l'implication des choix architecturaux qu'il pose (signification, cohérence, usage...).

\section{La cité des peintres}

Faisant écho, entre autres, à Utopia de Thomas Mores', aux unités d'habitation de Le Corbusier, à la ville générique de Rem Koolhaas, aux approches pédagogiques de Cédric Libert², aux Cités obscures dévoilées par François Schuiten et Benoît Peeters... un projet de cité des peintres en $U_{c h r o n i e^{3}}$ a été proposé aux étudiants.

Cette cité est constituée d'unités génériques traversées par des circulations (cf. figure 5). L'unité est un volume capable parallélépipédique de dimensions données $(6 \times 6 \times 15 \mathrm{~m})$ dans lequel quatre percements de section carrée sont imposés (2,4 $\mathrm{m}$ de côté), ils connectent les différentes unités entre elles (cf. figure 2). Par soustractions volumiques successives, l'étudiant inscrit son projet dans le parallélépipède en veillant à ce que les parois extérieures conservent une épaisseur minimale de $30 \mathrm{~cm}$. Le projet
1 - Le mot utopie vient du grec ou (ne pas) et topos (le lieu), il désigne donc littéralement un lieu qui n'existe pas. Certains voient dans le $u$ initial un eu grec, le mot a alors le sens de "lieu bon" ou de "lieu excellent." L'Utopic est le nom d'un pays imaginaire créé par Thomas More dans Utopia (1516)

2 - Voir à ce propos la conférence donnée par Cédric Libert au

Musée de la civilisation à Québec le 6 octobre 2011 dans le cadre des Instantanés d'architecture 20112012 de l'École d'architecture de l'Université Laval sur http://vimeo.com/32712676

3 - Le philosophe français Charles Renouvier a défini le concept d'uchronie à partir de celui d'utopie dans Uchronie : L'utopie dans l'histoire (1876). Sur le modèle du mot utopie, le mot uchronie vient des racines grecques ou (ne pas) et chronos (le temps). Le caractère uchronique d'un fait présuppose une liberté

d'interprétation et une contingence de l'histoire. Dès qu'une personne cherche "ce qui serait arrivé si..., elle déplace son discours en Uchronie.

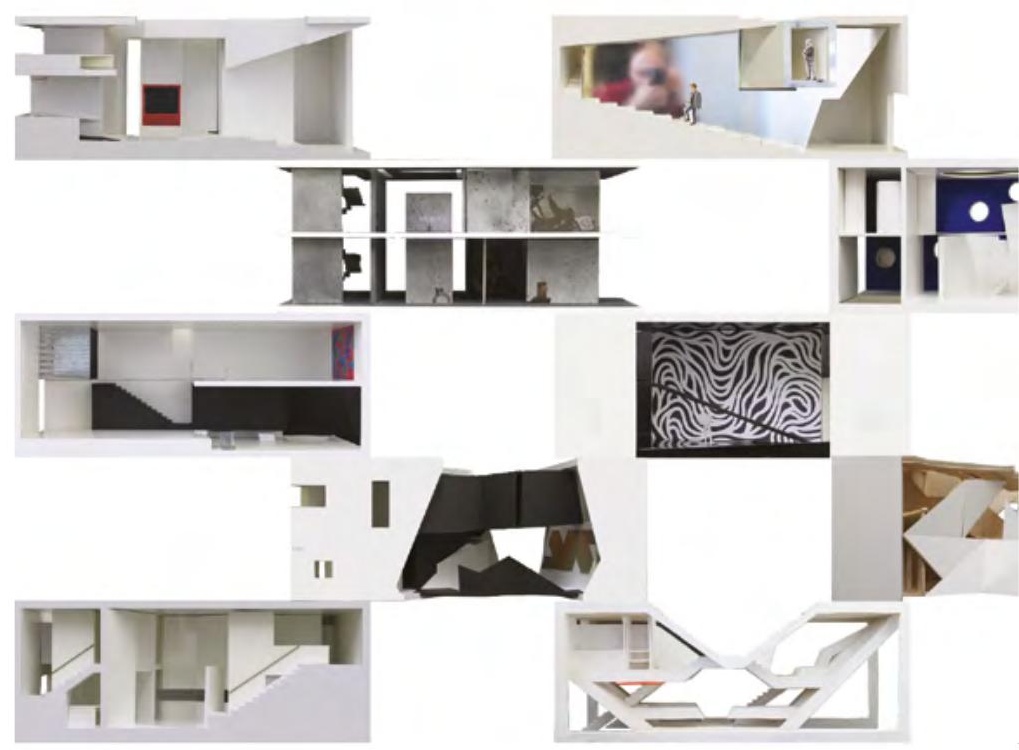


consiste en l'aménagement d'un atelier d'artiste, dont l'étudiant aura préalablement choisi un tableau. Celui-ci comprend : un espace atelier, un espace d'exposition, un sanitaire, un rangement de matériel, un point d'eau pour nettoyer les pinceaux, un lieu de collation, ainsi que la prise en compte des circulations intérieures/extérieures.

À la fin du projet, une cinquantaine de travaux d'étudiants sont sélectionnés consécutivement à la remise. Les maquettes de ces projets ont permis de reconstituer la cité des peintres lors d'une exposition (cf. figure 3 ).

\section{Retours de Bruxelles}

Tout en s'inscrivant dans la continuité d'autres projets proposés lors des années précédentes, cet énoncé remanié permet aux étudiants d'appréhender plus finement certaines questions architecturales, ce qui enrichit les performances pédagogiques et propédeutiques du projet.

1/ Les relations entre trois échelles spatiales sont interrogées puisque l'étudiant doit constamment équilibrer celles de la cité, de l'unité et de la pièce. II doit donc à la fois contextualiser son projet (une unité) à partir d'un environnement qui le dépasse (la cité) et le développer pour qu'il puisse, à son tour, être le contexte de mise en place des pièces qui le constituent. L'étudiant développe ainsi davantage son esprit critique et positionne directement sa réflexion dans un contexte plus vaste, il travaille sur un projet qui fait partie d'un ensemble et dont les contraintes prennent un sens lorsqu'il observe ses pièces à l'échelle de l'unité ou son unité à l'échelle de la cité.

2/ Les différents usages propres aux trois échelles mobilisent l'étudiant sur le caractère privatif des lieux. Différents types d'usagers parcourent et habitent la cité. Une unité peut être traversée par des artistes d'autres unités ou par de simples promeneurs. L'étudiant doit donc développer le rapport subtil entre le privé et le public, entre son unité et la cité, ou même au sein de l'unité entre les pièces. II étudie alors le rythme, la taille, l'épaisseur et la qualité des ouvertures et des vues qu'il projette dans son volume. Des questions telles que le vivre ensemble, le grand nombre ou la densité peuvent ainsi être appréhendées.

3/ Une incertitude relative propre au contexte de la cité met en tension tout projet d'unité. Si l'unité parallélépipédique constitue à priori un microcosme bien défini, le hasard et l'incertitude surgissent dans le projet de l'étudiant dès que celui-ci prend conscience qu'il ne peut prévoir quelles seront les unités qui vont l'entourer lors de leur assemblage dans la cité des peintres. Pas plus qu'il ne peut prévoir les circulations transversales d'usagers en promenade dans la cité et susceptibles de traverser certaines unités pour en rejoindre d'autres. 4/ Le rapport entre un volume capable, aux dimensions figées, et des espaces fonctionnels internes, aux tailles variées et initialement indéterminées, est difficile à gérer. Au départ, les dimensions exactes et la géométrie simple de l'unité paraissent rassurantes pour les étudiants débutants dont le premier réflexe est bien souvent de diviser l'espace pour l'organiser. Mais ils se retrouvent inévitablement confrontés à la question de la globalité, seule issue véritable au rétablissement de l'équilibre précaire à organiser "le plan comme une société de pièces" ${ }^{\prime \prime}$.

5/ La nature des circulations et des enveloppes est questionnée. L'étudiant gère les circulations internes à son unité, entre/dans les pièces, mais il doit

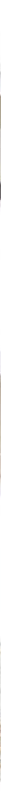


également gérer celles qui permettent les échanges entre l'unité et la cité. La qualité de ces échanges s'exprime pleinement dans le travail des limites intérieures des pièces et de l'enveloppe extérieure de l'unité. Lorsque toutes les unités sont assemblées, aléatoirement, pour former la cité, de nouveaux parcours émergent au gré des raccourcis ou des chemins de traverses créés par les étudiants.

Cet énoncé remanié permet également aux enseignants une mise en situation pédagogique différente puisqu'au-delà d'un projet individuel classique, celuici devient un projet d'atelier. L'atelier devient fédérateur par essence et son organisation table sur l'émulation collaborative du groupe plutôt que sur la réception individuelle d'un enseignement. Les craintes de certains enseignants quant à l'augmentation du nombre de contraintes prises en compte lors de ce type de projet ont été apaisées grâce aux bons résultats des étudiants. Cette réussite démontre qu'il est possible d'aborder des questions complexes, bien balisées par les enseignants, dès le premier quadrimestre de la première année de bachelier.

\section{Retours de Barcelone}

Une particularité du projet présenté ici est d'avoir réuni le temps d'un exercice des étudiants de deux écoles d'architecture. Après analyse des objectifs pédagogiques et détermination des convergences de programmes, il nous est apparu que le contenu des formations et les acquis des étudiants des deux institutions étaient relativement similaires. De plus, les contingences de planning et les modalités liées à la mobilité enseignante étaient favorables à notre entreprise.
À l'issue de la phase d'analyse, trente scénarios ont été sélectionnés à Bruxelles et transmis à Barcelone, aux étudiants de l'Escola Tècnica Superior d'Arquitectura del Vallès (Etsav). Sachant que des scénarios seraient sélectionnés pour servir de points de départ à des projets menés à Barcelone, les étudiants de Bruxelles ont accordé une attention toute particulière à la qualité de la dimension communicative de leur présentation.

La deuxième phase de l'exercice a été réalisée parallèlement à Bruxelles et à Barcelone, puisque trente projets y ont été réalisés à partir des scénarios mis en commun.

Alors que Bruxelles a considéré le projet en deux phases dont la première a fait l'objet d'une remise intermédiaire l'énoncé de la deuxième phase du projet a été pratiquement identique à Bruxelles et à Barcelone. Si ce n'est qu'à Barcelone l'exercice a été divisé en huit micro exercices d'une durée d'un jour ou deux. Les étudiants ont ignorés jusqu'à la dernière étape du projet l'objet final de l'exercice, l'atelier du peintre. Ils ont conçu l'atelier en séquences, petit à petit, sans être conscients de la globalité. L'introduction de l'exercice donnée par un enseignant de Bruxelles a permis aux étudiants de Barcelone de se confronter à un nouveau type de contenu et de méthodologie. Ils ont été immédiatement conscients de la dimension internationale de l'exercice et de l'apprentissage qu'ils pouvaient en tirer.

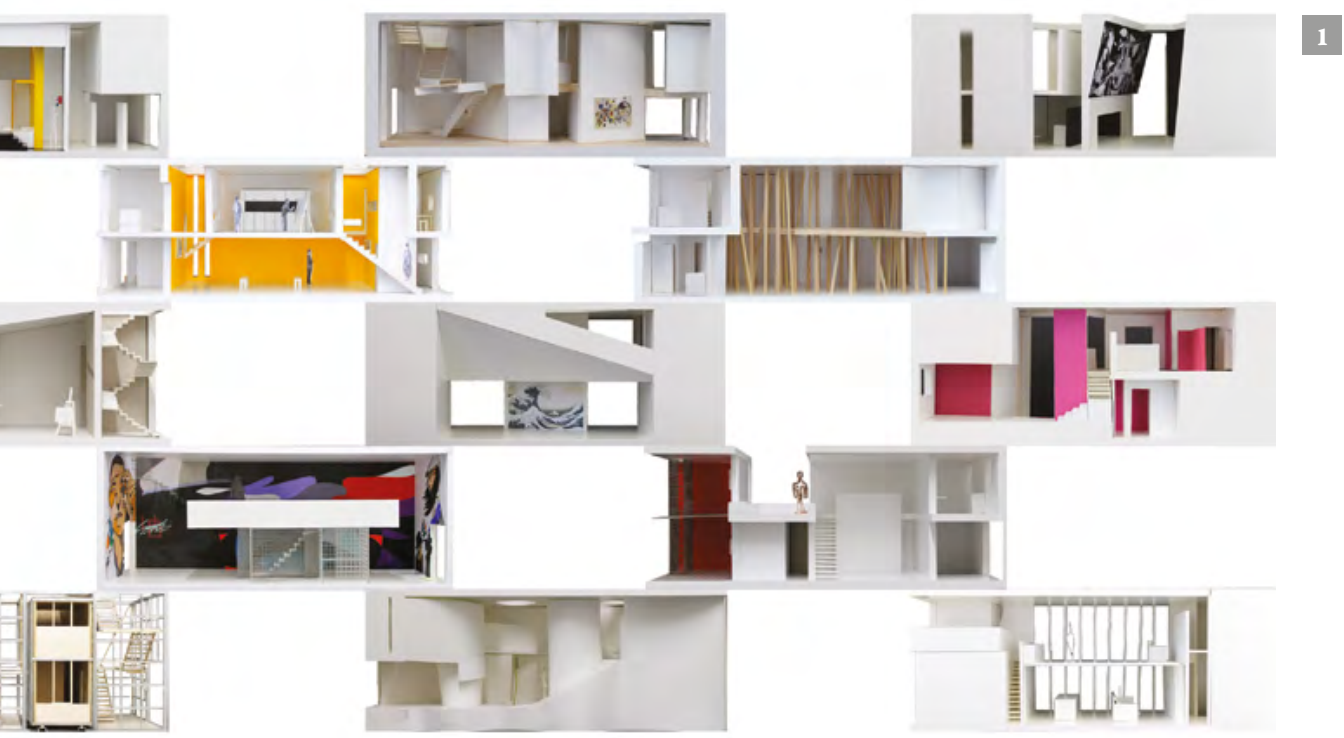

- La Cité des peintres à Bruxelles (pho tos et montage: Thierry Delcommune et Jean-Jacques Jungers). 


\section{Vers un exercice international et/ou intersites en bac 1 ?}

Le réel apport de la dimension internationale de cet exercice pose question. Force est de constater que la production des étudiants de Bruxelles et de Barcelone est encore à ce jour inconnue des uns et des autres, un blog sera réalisé à cet effet en collaboration avec l'université barcelonaise ${ }^{5}$ mais la distance ainsi que le nombre important d'étudiants en première année rend difficile un réel échange. Si nous décidons de poursuivre dans cette voie, une réflexion importante devra être menée en collaboration avec les commissions de programme et internationale de notre faculté bénéficiant d'une importante expertise dans le domaine afin de déterminer de quelle manière cette entreprise peut bénéficier davantage aux étudiants. Ce qui nous amène à nous demander si cet essai ne pourrait être transformé, à l'avenir, en collaboration avec les deux autres sites de notre faculté ? Sans présenter les difficultés logistiques précitées, un exercice commun aux trois sites dès le début de leur formation permettrait aux étudiants - ainsi qu'à leurs enseignants - de rencontrer leurs alterego en favorisant les échanges à venir et en tirant parti du caractère multi-site de notre belle faculté !

Auteurs de projets illustrés dans la figure 1 :

Madeleine Accarain | Morgane Blesch | Sixtine Bodard | Sandrine Borgniet | Caroline Creyf | Roselyne Deprez | Dorine Dispa | Henri de Fonvent | Ludovica De Gaudenzi | Stéphanie Charles de la Brousse | Alice Di Pompeo | Céline Ghanaï | Corentin Gillet | | Mathias Gommier | Emile de Hemptinne | Elliott Housiaux | Victor Kisielewicz | Gilles Libert | Roland Mangin | Adrien Mangon | Camille Matthews | Félix Mayaux | Grégoire Nelis | Juliette Poësson | Valentine Prouff | Katarzyna Skorowska | Cécile Struvay | Jean-Baptiste Tribolet | Xavier Van Bladel | Margaux Vande Berg | Louis Vierin | Timothy Walla | Manon Zizi

- Lunité générique : volume capable donné, zones de superposition en vert et zones de percements déterminés en rouge (Benoit Cruysmans et Jean-Jacques Jungers).

3-La Citédespeintres à Bruxelles (photo: Damien Claeys)

4- La Cité despeintres à Barcelone (photo: Anna Puigjaner).

5- La cité des peintres : assemblage des maquettes et circulation de base (Benoit Cruysmans et Jean-Jacques Jungers)

5- Voir le blog en ligne sur http:// cursintroducciol3-14.blogspot. com.es
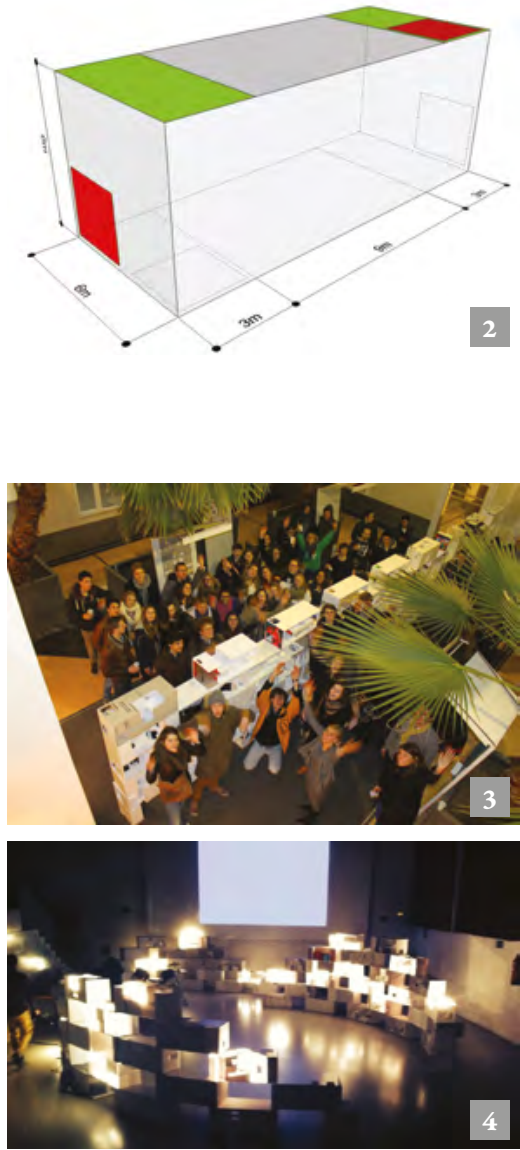

Enseignants du projet :

Marie-Christine Raucent (coordinatrice) | JeanLuc Capron | Francesco Cipolat | Damien Claeys | Benoît Cruysmans | Thierry Delcommune | Jean-Jacques Jungers | Gérald Ledent Michèle De Myttenaere | Michel Procès | JeanDenis Thiry | Pascale van ve Kerchove | David Vandenbroucke | Cécile Vandernoot

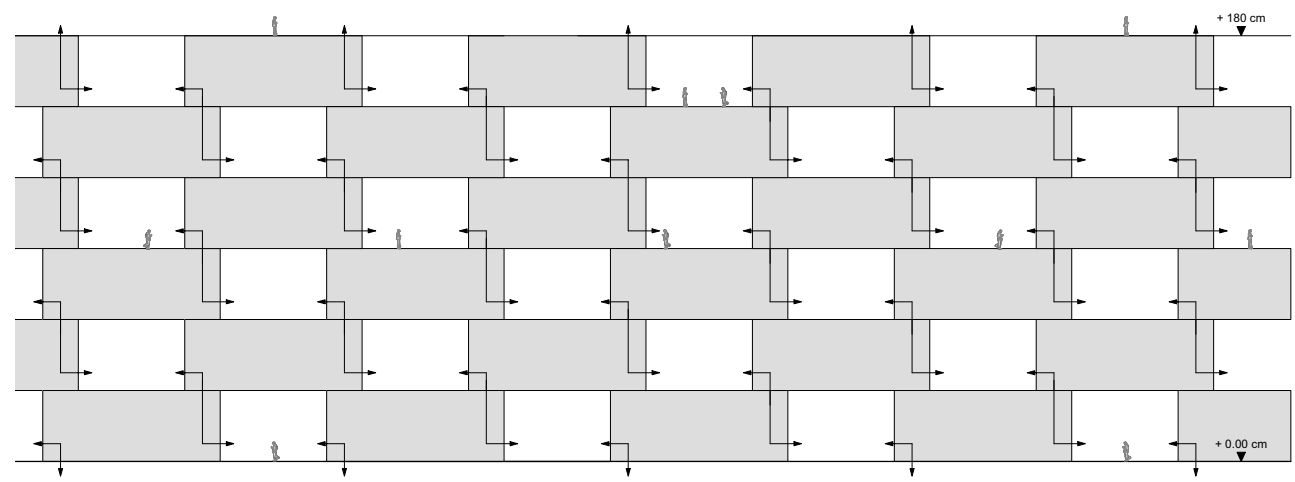

